\title{
Comparison of randomized and non- randomized controlled trials evidence regarding the effectiveness of workplace exercise on musculoskeletal pain control
}

\author{
RFC Moreira ${ }^{\mathrm{a},{ }^{*}}$, FA Foltran ${ }^{\mathrm{a}}, \mathrm{F}$ Albuquerque-Sendín ${ }^{\mathrm{b}}$, MC Mancini ${ }^{\mathrm{c}}$ and HJCG Coury ${ }^{\mathrm{a}}$ \\ ${ }^{a}$ Physical Therapy Department, Federal University of São Carlos, Rodovia Washington Luis, Km 235. Zip Code \\ 13565-905, São Carlos, São Paulo, Brazil \\ ${ }^{\mathrm{b}}$ Physical Therapy Department, University of Salamanca, Donante de Sangre $s / n$ - Campus "Miguel de \\ Unamuno", Zip Code 37007, Salamanca, Spain \\ ${ }^{\mathrm{c}}$ Occupational Therapy Department, Federal University of Minas Gerais, Av. Antonio Carlos, 6627 - Campus \\ Universitario Pampulha, Zip Code 31270-010, Belo Horizonte, Minas Gerais, Brazil
}

\begin{abstract}
Evidence synthesized based on randomized controlled trials (RCT) results are recognized as the pinnacle of research excellence; however, the conduction of RCT in workplace environment is not always possible. This study comparatively reviewed evidence from RCT and non-RCT studies in which participants performed workplace exercise for musculoskeletal pain control. Up to February 2011, PubMed, MEDLINE, Embase, Cochrane, PEDro and Web of Science databases were searched. All trials that evaluated workplace exercise interventions for controlling musculoskeletal pain were included. The PEDro scale was used to rate the studies' quality, PRISMA and Cochrane recommendations were applied, and association between frequencies of effect size categories (small, moderate, large) from various outcomes by study type was tested ( $2 \times 3$ contingency table). The search yielded 10239 references in English, from which 21 RCT and 12 non-RCT were selected. Both groups of studies presented methodological flaws including descriptions of randomization, blinding of examiners and absence of intention-totreat analysis for the RCT, and further absence of controls and blind assessor for the non-RCTs. RCTs had significantly more moderate and large effect size reported in their results compared to non-RCTs $(\mathrm{p}=0.04)$. Considering the difficulties in randomizing participants in occupational settings, all studies would benefit from better describing pertinent methodological information.
\end{abstract}

Keywords: physical education and training; workers; prevention, musculoskeletal diseases, evaluation

\section{Introduction}

Musculoskeletal disorders have been recognized as a worldwide health problem. One of the measures for controlling these disorders is workplace exercise practice. However, besides being frequently used for musculoskeletal pain and disorders control, there is controversy regarding effectiveness and the means of implementation of such intervention.

In spite of representing the pinnacle of research excellence, randomized clinical trial designs are not always possible, adequate or ethical $[6,34,39]$ and its implementation in natural settings, as the occupational ones, is not simple. Several authors have described practical factors that substantially limit the opportunities for conducting RCTs to foster occupational safety and health promotion programs $[4,6,39,50]$. Among them, employees usually work in groups making it impossible to change work conditions or behavior individually.

Considering these difficulties, quasi-experimental designs involving comparison groups and pre-post

*Corresponding author. E-mail: roberta.carreira@gmail.com. 
studies are often conducted in occupational settings. However, according to Silverstein and Clark [39] these studies can only be considered effective when they adequately measure exposure, health outcomes and potential confounders or effect modifiers.

External validity is also important to be evaluated as it can threat the possibility of the results to be generalized to other groups, time and settings [14].

Taking into account the advantages and difficulties of conducting RCT in the workplaces, the present study comparatively reviewed evidence from randomized and non-randomized controlled trials (non RCT) in which participants had performed exercise at worksites for musculoskeletal pain control. So, the current review aims to evaluate the methodological aspects and results from systematically reviewed RCT and non RCT in order to verify their potential problems and contributions to improve workplace preventive interventions based on exercises. Besides the specific context of the present review, the methodological criteria adopted here tried to follow whenever possible the recommendations proposed by PRISMA [29] and Cochrane [21] collaborations for systematic review conduction.

\section{Materials and methods}

\subsection{Literature search strategy}

Up to February, 2011 a search on PubMed, MEDLINE, Embase, Cochrane, PEDro, and Web of Science databases was conducted using the following keywords: workplace, musculoskeletal diseases, occupational diseases, musculoskeletal complaints, symptoms, exercise, preventive exercise, worksite physical activity, warming up, stretching, break rest, work pause, ergonomic intervention, ergonomic training, ergonomic program, efficacy, effectiveness, evaluation. Each electronic database was searched from the earliest year available to identify relevant studies published in the English language. Two independent reviewers (RFCM and FAF) selected the studies based on 3 consecutive phases: 1 . title selection; 2. abstract review and 3. full paper retrieval and review to identify those which match the inclusion criteria regarding type of study design, participants, intervention and outcomes. Reviewers independently selected the trials to be included in the review using a standard form adapted from the Cochrane Collaboration. Disagreements during the whole process were solved by consensus.

\subsection{Eligibility criteria for initial study selection}

\subsubsection{Type of study}

Randomized controlled trials and quasiexperimental designs with comparison groups, and pre-post type prospective studies were eligible to be included in this study.

\subsubsection{Participants}

Only studies reporting results from active working population at their current occupational activities were analyzed.

\subsubsection{Types of interventions}

Trials either investigating or comparing workplace interventions including exercise for musculoskeletal symptoms prevention were selected.

\subsubsection{Outcome measures}

Studies investigating musculoskeletal symptoms, particularly pain, as one of the main outcome measures were included.

\subsection{Methodological quality assessment of the in- cluded studies}

The PEDro scale [49] was used to rate the quality of both groups of studies included in this review: RCT and non RCT. PEDro scale was the eligible methodological evaluation tool as it covers the four main types of bias pointed by the Cochrane Collaboration (the more important agency for providing recommendations for health care interventions): 1 . selection bias; 2. performance bias; 3 . attrition bias and 4. detection bias[21], which may threat the internal validity of both RCT and non RCT studies.

Each PEDRo's criterion is scored according to its presence or absence in the evaluated study. The criteria assessed are related to: 1. Specification of eligibility criteria; 2. Random allocation of the subjects to groups; 3. Concealment of allocation; 4. Similarity of the groups at baseline regarding the most important prognostic indicators; 5. Blinding of all subjects; 6 . Blinding of all therapists who administered the therapy; 7. Blinding of all assessors who measured at least one key outcome; 8. Measurement of key outcomes for more than $85 \%$ of the subjects initially allocated to groups; 9. Inclusion of "intention to treat" analysis for at least one key outcome; 10. Report of between-group statistical comparisons for at least one key outcome; 11. Report of both point measures and measures of variability for at least one 
key outcome. Each satisfied item (except the first) contributes one point to the total score (range $=0-10$ points).

The external validity was independently evaluated based on 4 criteria previously adopted by van Poppel et al [48]: 1. Homogeneity of participants; 2. Adequate control group; 3. Relevant outcome measures; 4. Follow up longer than 6 months.

\subsection{Data extraction}

All authors independently extracted data regarding study design, study participants, comparison groups, intervention performed (sample sizes, type of exercise performed, frequency and duration of each session), outcome measures, evaluation tools and outcomes using a standardized form. Information from group(s) mean(s) and standard deviation(s) for each outcome was obtained from RCT and non RCT studies.

\subsection{Effect size calculation}

Effect sizes (ES) from studies that provided the required information were calculated using the software $G^{*}$ Power $3.1 \AA$. Studies that conducted both within-group and between-group comparisons, had effect sizes calculated only for the between-group comparison from post-intervention measurement(s). ES were further classified as small, moderate or large, according to Cohen's criteria [8] for standardized differences in means, using the thresholds 0.20 , 0.50 and 0.80 , respectively.

\subsection{Data analysis}

The data collected through the standardized form were descriptively analyzed. After this, nominal data for each PEDro scale criteria, external validity, items related to exercise protocols reproducibility, number and type of pain evaluation tools were categorized as sufficient or not sufficient according to its description and compared by the Exact Fisher test. Also, 2 (RCT, non RCT) x 3 (ES category) contingency table tested association between frequency of ES and study type. Total PEDro score for RCT and non RCT studies were compared by Mann Whitney U test. These analyses were conducted with the SPSS 19.0 package SPSS, Chicago, IL) with level of significance $\alpha=0.05$.

\section{Results}

The electronic search yielded a total of 10239 references published in English. At the end of the selection process, $21 \mathrm{RCT}$ and 12 non RCT satisfied the inclusion criteria and were included in the present review. From the 12 non RCT retrieved, two studies were classified as double publication for musculoskeletal pain outcomes [35,38] lasting 11 studies for drawing conclusions (Table 1). From the 21 RCTs, one was a double publication [2,7] lasting 20 RCTs for the final analysis (Table 2). Overall, $50 \mathrm{ES}$ were calculated, from those, 40 ES were from 17 RCT $[1,3,15,18-20,22,24,25,30,33,41,42,44-47]$, and 10 ES from 4 non RCT studies $[17,35,36,43]$. The remaining 3 RCT [2,27,37] and 7 non RCT $[5,12,13,16,28,31,40]$ did not provide information necessary for ES calculation.

\subsection{Internal validity}

The results of internal validity scores for non $\mathrm{RCT}$ and RCT studies are shown in Tables 1 and 2, respectively. Taking into account the maximum score possible for the PEDro scale (10 points), the non randomized studies group achieved a mean score of $2.09 \pm 0.92$ points $(\min 1 ; \max 4)$, while the RCT group achieved a mean value of $4.65 \pm 1.18$ points (min3; max8). However, according to Maher [32], due to the impossibility of achieving conditions such as blinding of therapist (criterion 5) or subjects (criterion 6) in clinical trials conducted in the workplace, the maximum score that can be reached by these clinical trials is $8 / 10$. For the non RCT studies, another two criteria are not possible to be achieved due to the own nature of this study group: random allocation (criterion 2) and concealed allocation (criterion 3). In this case, the maximum score that can be reached by the non RCT group is $6 / 10$. These limitations suggested the need for a data adjustment according to the maximum possible score for each group, i.e, 6 for non RCT and 8 for the RCT group. When considering the adjusted analyses, a mean relative score of $0.35 \pm 0.19$ ( $\min 0.17$; $\max 0.67)$ was achieved for the non RCT group, while a mean relative value of $0.58 \pm 0.15$ (min $0.37 ; \max 1)$ was reached for the RCT group. These results represented a mean percentage of $35 \%$ of the maximum score possible for the non RCT group and $58 \%$ for the RCT group.

Significant difference were identified between RCT and non RCT groups for both PEDro total $(p<0.001)$ and PEDro relative $(p=0.003)$ scores. 
The comparison between RCT and non RCT studies for each PEDro criterion analysis showed significant differences between groups for four PEDro scale criteria assessed. Fisher test identified significant differences for the following criteria: (1) Specification of eligibility criteria $(\mathrm{p}=0.001)$; (2) Random allocation of subjects to groups $(\mathrm{p}<0.001)$; (4) Similarity of the groups at baseline ( $p=0.047)$ and; (11) Report of point and variability measures for at least one key outcome $(\mathrm{p}=0.042)$. Besides Fisher test having not identified significant difference for both criteria 8 - Measurement of at least one key outcome for more than $85 \%$ of subjects initially allocated to groups, and criteria 9 - Description of an intention to treat analysis; both of them were observed in $40 \%$ of the RCT and only in $18 \%$ of the non RCT studies. Despite these discrepancies, Fisher test did not revealed significant difference, probably due to the small number of non RCT studies included.

Table 1

Assessment of methodological quality of non RCT studies by PEDro scale

\begin{tabular}{|c|c|c|c|c|c|c|c|c|c|c|c|c|c|}
\hline \multirow[b]{2}{*}{ Study } & \multicolumn{13}{|c|}{ PEDro Scale } \\
\hline & 01 & 02 & 03 & 04 & 05 & 06 & 07 & 08 & 09 & 10 & 11 & $\begin{array}{l}\text { PEDro } \\
\text { Score }\end{array}$ & $\begin{array}{l}\text { Relative } \\
\text { PEDro } \\
\text { score* }\end{array}$ \\
\hline Genaidy et al. [17] & - & - & - & - & - & - & - & - & - & + & + & 2 & 0.33 \\
\hline Balci \& Aghazadeh [5] & - & - & - & - & - & - & - & - & - & + & - & 1 & 0.17 \\
\hline Mongini et al [35] & - & - & - & - & - & - & - & + & + & + & + & 4 & 0.67 \\
\hline Mongini et al [36] & - & - & - & - & - & - & + & - & + & + & + & 4 & 0.67 \\
\hline Macedo et al [31] & - & - & - & - & - & - & - & - & - & + & + & 2 & 0.33 \\
\hline Fenety and Walker [16] & + & - & - & - & - & - & - & + & - & - & + & 2 & 0.33 \\
\hline Dehlin et al. [13] & - & - & - & - & - & - & - & - & - & + & - & 1 & 0.17 \\
\hline Dehlin et al. [12] & - & - & - & - & - & - & - & - & - & + & - & 1 & 0.17 \\
\hline Shinozaki [40] & - & - & - & - & - & - & - & - & - & + & - & 1 & 0.17 \\
\hline Skargren and Oberg [43] & + & - & - & + & - & - & - & - & - & + & - & 2 & 0.50 \\
\hline Leclerc et al . [28] & + & - & - & - & - & - & - & - & - & + & + & 2 & 0.33 \\
\hline $\begin{array}{l}\text { Number of studies which } \\
\text { satisfied the PEDro criteria }\end{array}$ & 3 & 0 & 0 & 1 & 0 & 0 & 1 & 2 & 2 & 10 & 7 & & \\
\hline
\end{tabular}

*Values calculated in function of the maximum score possible for non randomized controlled trials group: 6 points.

The index used was: relative PEDro score=total PEDro score/6

Table 2

Assessment of methodological quality of RCT studies by PEDro scale

\begin{tabular}{|c|c|c|c|c|c|c|c|c|c|c|c|c|c|}
\hline \multirow[b]{2}{*}{ Study } & \multicolumn{13}{|c|}{ PEDro Scale } \\
\hline & 01 & 02 & 03 & 04 & 05 & 06 & 07 & 08 & 09 & 10 & 11 & $\begin{array}{l}\text { PEDro } \\
\text { Score }\end{array}$ & $\begin{array}{l}\text { Relative } \\
\text { PEDro } \\
\text { score** }\end{array}$ \\
\hline Donchin et al. [15] & + & + & - & + & - & - & - & - & + & + & - & 4 & 0.5 \\
\hline Kellett et al. [24], 1991 & + & + & - & + & - & - & - & - & - & + & + & 4 & 0.5 \\
\hline Groningsater et al [19] & + & + & - & + & - & - & - & + & - & + & + & 5 & 0.6 \\
\hline Gundewall et al. [20] & - & + & - & - & - & - & - & + & - & + & + & 4 & 0.5 \\
\hline Takala et al [44] & + & + & - & + & - & - & + & + & - & + & + & 6 & 0.8 \\
\hline Gerdle et al. [18] & + & + & - & - & - & - & + & - & - & + & + & 4 & 0.5 \\
\hline
\end{tabular}




\begin{tabular}{|c|c|c|c|c|c|c|c|c|c|c|c|c|c|}
\hline Lundblad, et al. [30] & + & + & - & + & - & - & - & - & - & + & + & 4 & 0.5 \\
\hline Ahlgren et al. [1] & + & - & + & - & - & - & - & - & - & + & + & 4 & 0.5 \\
\hline Horneij et al. [22] & + & + & - & - & - & - & - & - & + & + & + & 4 & 0.5 \\
\hline Larsen et al. [27] & + & + & - & + & - & - & - & + & + & + & + & 6 & 0.8 \\
\hline van den Heuvel et al. [47] & + & + & - & - & - & - & - & - & - & + & + & 3 & 0.4 \\
\hline Tsauo et al. [45] & - & + & - & - & - & - & - & - & - & + & + & 3 & 0.4 \\
\hline Maul et al. [33] & + & + & - & + & - & - & - & - & - & + & + & 4 & 0.5 \\
\hline Sjogren et al. [41] & + & + & + & + & - & - & + & + & + & + & + & 8 & 1.0 \\
\hline Sjogren et al. [42] & + & + & - & - & - & - & - & + & + & + & + & 5 & 0.6 \\
\hline Kietrys et al. [25] & + & + & - & - & - & - & - & + & + & + & + & 5 & 0.6 \\
\hline Andersen et al. [2] & + & + & - & - & - & - & + & + & + & + & + & 6 & 0.8 \\
\hline Andersen et al [3] & + & + & - & + & - & - & - & - & - & + & + & 4 & 0.5 \\
\hline Tveito and Eriksen [46] & + & + & + & + & - & - & - & - & - & + & + & 5 & 0.6 \\
\hline Pedersen et al. [37] & + & + & - & - & - & - & + & - & + & + & + & 5 & 0.6 \\
\hline $\begin{array}{l}\text { Number of studies which } \\
\text { satisfied the PEDro crite- } \\
\text { ria }\end{array}$ & 18 & 19 & 3 & 10 & 0 & 0 & 5 & 8 & 8 & 20 & 19 & & \\
\hline
\end{tabular}

**Values calculated in function of the maximum score possible for randomized controlled trials group: 8 points.

The index used was: relative PEDro score $=$ total PEDro score $/ 8$.

\subsection{External validity}

The comparison between groups through the Fisher test showed significant differences for the homogeneity $(\mathrm{p}<0.001)$ and adequate control group $(p<0.001)$ criteria. These two criteria were satisfied by $90 \%$ of the RCT studies, but were met by only $18 \%$ of the non RCT ones. Regarding the report of relevant results (criteria 3 ), $35 \%$ of RCTs and $45 \%$ of the non RCT satisfied this criteria $(p=0.705)$. Regarding the last validity criteria evaluated (follow up longer than 6 months) the groups presented very similar results: $25 \%$ of RCT and $27 \%$ of non RCT met the criteria $(\mathrm{p}>0.9)$.

\subsection{Training protocol description}

Significant differences were identified for type of exercise applied and for the frequency and duration of exercise sessions. Each item was classified either as insufficient or sufficient, according to its in/adequate description. Table 3 present the descriptive and statistics results related to the protocols adopted in both groups.
Table 3

Comparison of protocol characteristics

\begin{tabular}{lccc}
\hline & \multicolumn{2}{c}{$\begin{array}{c}\text { Absolute number and percentage of studies with } \\
\text { sufficient }\end{array}$} \\
\hline $\begin{array}{l}\text { Study } \\
\text { Type }\end{array}$ & $\begin{array}{c}\text { Body } \\
\text { region }\end{array}$ & $\begin{array}{c}\text { Type of } \\
\text { exercise }\end{array}$ & $\begin{array}{c}\text { Frequency and dura- } \\
\text { tion of the session }\end{array}$ \\
\hline RCT & $16(80 \%)$ & $20(100 \%)$ & $20(100 \%)$ \\
Non RCT & $7(63 \%)$ & $8(72 \%)$ & $7(63 \%)$ \\
$\begin{array}{l}\text { p value } \\
\text { (Fisher } \\
\text { test) }\end{array}$ & 0.405 & $0.037 *$ & $0.01 *$ \\
\hline
\end{tabular}

* Statistical significant difference $(\mathrm{p}<0.05)$

\subsection{Symptoms evaluation}

The effects of exercise intervention on pain results were mainly assessed by questionnaires in both groups. However, while $65 \%$ of the RCT studies used two or more pain evaluation tool to assess variables related to pain, only $27 \%$ of the non RCT study group has performed a more comprehensive analysis of this symptom using 2 or more objective evaluation tools $(\mathrm{p}=0.066)$. 


\subsection{Effect size by study type}

A significant association between ES category and study type was found $(\mathrm{p}=0.0400)$, with $\mathrm{RCT}$ s reporting greater frequencies of moderate and large ES and non RCTs reporting small ES (Table 4).

Table 4

Frequency distribution of Effect Size category by Study type

\begin{tabular}{lccc}
\hline Study & \multicolumn{3}{c}{ Effect Size (ES) Category } \\
\cline { 2 - 4 } Type & Small & Moderate & Large \\
\hline RCT & 16 & 15 & 9 \\
Non RCT & 8 & 0 & 2 \\
\hline
\end{tabular}

\section{Discussion}

The great heterogeneity regarding methodological quality of the primary studies in RCT and non RCT studies could be identified as the main reason that precluded clinical evidence synthesis and comparison between the RCT and non RCT groups. On the other hand important scientific recommendations could be synthesized regarding future research on exercise workplace exercise interventions.

The assessment of internal validity by the PEDro scale revealed low methodological quality of non randomized studies performed in the workplace for musculoskeletal pain prevention. After adjusting the data to the maximum score per group, the non randomized studies presented a low mean score (less than $50 \%$ of the maximum score possible), while the RCT group achieved a mean value of $58 \%$ of the total maximum score possible. Thus, comparatively, both groups presented expressive methodological problems indicating the need for improvements, in order to provide their contribution for interventions aiming at controlling pain in occupational settings. This is particularly valid for the non RCT that, despite the absence of randomization, have presented extra deficiencies, as the common absence of control groups and blind assessor, which threat the internal validity of the studies and contribute to biased results [23]. Regarding the RCT studies, the main deficiencies identified were inadequate descriptions of randomization procedures, blinding of examiners and absence of intention-to-treat analysis. The absence of examiners' blinding has been pointed out as one of most important features to increase the potential for biases [26]. Thus, despite the fact that randomized controlled trials have been often cited as the most powerful design in research evidence for evaluating health care effectiveness [11], they can also present considerable methodological limitations.

In general, the external validity was low for both groups of studies. However, the RCT showed better results for homogeneity and adequate control of group. Both groups of studies would benefit from better reporting relevant results and performing longer follow ups. Comparatively, the non RCT studies presented poorer descriptions of the exercise protocols. This insufficient description reduces the possibilities of evaluating these protocols, their results and reproducibility Furthermore, the RCT studies included more tools for evaluating the main outcome. Considering the challenge still involved in the evaluation of pain in occupational settings, and absence of a consensual methodology for this task [10], the RCT studies seems to have more chances of fulfilling a comprehensive evaluation.

This argument might also be related to the fact that while the RCTs provided strong evidence about the effectiveness of workplace exercise in controlling neck pain among workers who performed sedentary tasks, and moderate evidence for low back pain relief among workers who performed heavy physical tasks, as previously evaluated by Coury et al. [9]; the non RCT analyses did not allow drawing any substantial clinical conclusion. As reported earlier, stronger methodological flaws than just the lack of an adequate random allocation of the subjects between groups were presented. Furthermore, in addition to weaknesses in method procedures, non RCT tend not to report necessary information for effect size calculation. In fact, while $85 \%$ of the selected RCT provided information such as study groups means and standard deviations, only $36.4 \%$ of the non RCT provided such information. Also, the frequency of methodological flaws observed in non RCTs seems to be followed by results with effect sizes of smaller magnitudes, compared to RCTs.

The above-mentioned characteristics of non RCT studies clearly minimize their potential for contributing with practical workplace exercise evidence synthesis. The stronger methodological control of RCT studies may yield appropriate conditions for minimizing threats to internal validity and, consequently, offer conditions such that reports of intervention effects may be optimized. 


\section{Limitations}

The current review presents some weakness that need to be considered. The search strategy, restricted to English-language publications, could have contributed to reduce the number of potential studies included in each group. The low number of non RCTs studies identified and included here generated an unbalanced comparison between groups. Another point to be mentioned is the lack of standardized pain evaluation tool which jeopardized the studies comparability. However, this condition is related to the multidimensional nature of pain evaluation and, thus, beyond the scope of this review.

\section{Conclusion}

It would be expected that non-randomized trials would lead to lower methodological quality evaluation than randomized trials, particularly when the studies are assessed by protocols addressed to evaluate aspects related to randomization. However, the non-randomized trials included in this review have presented flaws greater than the ones related to the randomization process itself. Thus, considering the difficulties in applying the randomization design in interventions intended for collective situations in occupational settings, the programs would benefit from the authors providing detailed methodological information. The RCTs would be improved by the inclusion of better descriptions of randomization procedures, blinding of examiners and the intention-totreat analysis, while the non-RCTs would be improved by the inclusion of a control group and blind assessors.

\section{References}

[1] C. Ahlgren, K. Waling, F. Kadi, M. Djupsjöbacka, L.E. Thornell, G. Sundelin, Effects on physical performance and pain from three dynamic training programs for women with workrelated trapezius myalgia, Journal of Rehabilitation Medicine 33 (2001), 162-169.

[2] L.L. Andersen, M.B. Jørgensen, A.K. Blangsted, M.T. Pedersen, E.A. Hansen, G. Sjøgaard, A randomized controlled intervention trial to relieve and prevent neck/shoulder pain, Medicine \& Science in Sports \& Exerercise 40 (2008), 983990.

[3] L.L. Andersen, M. Kjaer, K. Søgaard, L. Hansen, A.I. Kryger, G. Sjøgaard, Effect of two contrasting types of physical exercise on chronic neck muscle pain, Arthritis \& Rheumatism 59 (2008), 84-91
[4] R. Baker, A. Brockhaus, D. Boucier, L. Chapman, J. Collins, L. Goldenhar, C. Heaney, T. Katz, P. Landsbergis, J. Martonik, I. Most, S. Schneider, T. Scharf, R. Sinclair, May 2000 Supplement on Preventing Occupational Injuries, American Journal of Preventive Medicine 20 (2001), 308-309.

[5] R. Balci and F. Aghazadeh, The effect of work-rest schedules and type of task on the discomfort and performance of VDT users, Ergonomics 46 (2003), 455-465.

[6] N. Black, Why we need observational studies to evaluate the effectiveness of health care, Britsh Medical Journal 312 (1996), 1215-1218.

[7] A.K Blangsted, K. Søgaard, E.A. Hansen, H. Hannerz, G. Sjøgaard, One-year randomized controlled trial with different physical-activity programs to reduce musculoskeletal symptoms in the neck and shoulders among office workers, Scandinavian Journal of Work, Environmental \& Health. 34 (2008), 55-65.

[8] J. Cohen, Statistical power analysis for the behavioral sciences. 2nd ed. Hillsdale, NJ: Lawrence Erlbaum Associates, 1988.

[9] H.J.C.G. Coury, R.F.C. Moreira, N.B.D. Dias. Evaluation of workplace exercise effectiveness on neck, shoulder and low back pain control: a systematic review. Revista Brasileira de Fisioterapia 13 (2009), 461-479.

[10]F. Coutarel, Symposia - Evaluation of interventions designed to prevent MSD: Theories and strategies, In: Seventh International Conference on Prevention of Work-Related Musculoskeletal Disorders, 2010, Angers, France. Book of Abstract PREMUS, Seventh International Conference on Prevention of Work-Related Musculoskeletal Disorders 2010, 44.

[11] A. Dechartres, P. Charles, S. Hopewell, P. Ravaud, D.G. Altman, Reviews assessing the quality or the reporting of randomized controlled trials are increasing over time but raised questions about how quality is assessed. Journal of Clinical Epidemiology 64 (2011), 136-144.

[12] O. Dehlin, S. Berg, G.B.J. Andersen, G. Grimby, Effect of physical training and ergonomic counseling on the psychological perception of work and on the subjective assessment of low-back insufficiency, Scandinavian Journal of Rehabilitation Medicine 13 (1981), 1-9.

[13] O. Dehlin, S. Berg, B. Hedenrud, G.B.J. Andersson, G. Grimby, Muscle training, phychological perception of work and low-back symptoms in nursing aides. Scandinavian Journal of Rehabilitation Medicine 10 (1978), 201-209.

[14]E. Domholdt, Research Validity. In: Physical Therapy Research - Principles and Applications, W.B Saunders Company, Philadelphia (PA), 2000, pp. 77-94.

[15]M. Donchin, O. Woolf, L. Kaplan, Y. Floman, Secondary prevention of low-back pain. A clinical trial, Spine 15 (1990), $1317-1320$

[16]A. Fenety and J.M. Walker, Short-term effects of worksatation exercises on musculoskeletal discomfort and postural changes in seated video display unit workers. Physical Therapy 82 (2002), 578-589.

[17]A.M. Genaidy, E. Delgado, T. Bustos, Active microbreak effects on musculoskeletal comfort ratings in meatpacking plants, Ergonomics 38 (1995), 326-336.

[18]B. Gerdle, C. Brulin, J. Elert, P. Eliasson, B. Granlund, Effect of a general fitness program on musculoskeletal symptoms, clinical status, physiological capacity, and perceived work environment among home care service personnel. Journal of Occupational Rehabilitation 5 (1995), 1-16.

[19]H. Gronningsaeter, K. Hytten, G. Skauli, C.C. Christensen, H. Ursin, Improved health and coping by physical exercise or cognitive behavioral stress management training in a work environment, Psychology and Health 7 (1992), 147-63. 
[20]B. Gundewall, M. Liljeqvist, T. Hansson, Primary prevention of back symptoms and absence from work. A prospective randomized study among hospital employees. Spine 18 (1993), 587-594.

[21] J.P.T. Higgins and S. Green, editors. Cochrane handbook for Systematic Reviews of Interventions 4.2.6 [update September 2006]. In: Cochrane Library, Chichester, UK: John Wiley \& Sons, Ltd., 2006.

[22]E. Horneij, B. Hemborg, I. Jensen, C. Ekdahl, No significant differences between intervention programmes on neck, shoulder and low back pain: a prospective randomized study among home-care personnel. Journal of Rehabilitation Medicine. 33 (2001), 170-176.

[23] A.R. Jadad, R.A. Moore, D. Carroll, C. Jenkinson, D.J. Reynolds, D.J. Gavaghan, H.J. McQuay, Assessing the quality of reports of randomized clinical trials: is blinding necessary?, Controlled Clinical Trials 17 (1996), 1-12.

[24] K.M. Kellett, D.A. Kellett, L. Nordholm, Effects of an exercise program on sick leave due to back pain, Physical Therapy. 71 (1991), 283-291.

[25]D.M. Kietrys, J.S. Galper, V. Verno, Effects of at-work exercises on computer operators, Work 28 (2007), 67-75.

[26] B.W. Koes and J.L. Hoving. The value of the randomised clinical trial in the field of physiotherapy. Manual Therapy 3 (1998), 179-86.

[27]K. Larsen, F. Weidick, Y de C, Leboeuf, Can passive prone extensions of the back prevent back problems? A randomized, controlled intervention trial of 314 military conscripts, Spine 27 (2002), 2747-2752.

[28]A. Leclerc, M.F. Landre, F. Pietri, M. Beaudoin, S. David, Evaluation of interventions for prevention of back, neck, and shoulder disorders in three occupational groups, International Journal of Occupational and Environmental Health, 3 (1997), $5-11$.

[29] A. Liberati, D.G. Altman, J. Tetzlaff; C. Mulrow; P.C. Gøtzsche, J.P.A. Ioannidis, M. Clarke, P.J. Devereaux, J. J. Kleijnen, D. Moher, The PRISMA statement for reporting systematic reviews and meta-analyses of studies that evaluate healthcare interventions: explanation and elaboration. British Medical Journal 2009;339:b2700.

[30]I. Lundblad, J. Elert, B. Gerdle, Randomized controlled trial of physiotherapy and feldenkrais interventions in female workers with neck-shoulder complaints, Journal of Occupational Rehabilitation 9 (1999), 179-194.

[31] A.C. Macedo, C.S. Trindade, A.P. Brito, M.S. Dantas, On the effects of a workplace fitness program upon pain perception: a case study encompassing office workers in a Portuguese context, Journal of Occupational Rehabilitation 21 (2010), 228233.

[32]C.G. Maher, A systematic review of workplace interventions to prevent low back pain, The Australian Journal of Physiotherapy 46 (2000), 259-269.

[33]I. Maul, T. Läubli, M. Oliveri, H. Krueger, Long-term effects of supervised physical training in secondary prevention of low back pain, European Spine Journal 14 (2005), 599-611.

[34] S. Milanese, The use of RCT's in manual therapy-are we trying to fit a round peg into a square hole?, Manual Therapy 16 (2011), 403-405.

[35]F. Mongini, G. Ciccone, E. Rota, L. Ferrero, A. Ugolini, A. Evangelista, M. Ceccarelli, C. Galassi, Effectiveness of an educational and physical programme in reducing headache, neck and shoulder pain: a workplace controlled trial, Cephalalgia 28 (2008), 541-552.

[36]F. Mongini, A. Evangelista, E. Rota, L. Ferrero, A. Ugolini, M. Ceccarelli, G. Ciccone, C. Galassi, Long-term benefits of an educational and physical program on headache, and neck and shoulder pain, in a working community, The Journal of Pain 10 (2009), 1138-1145.

[37]M.T. Pedersen, A.K. Blangsted, L.L. Andersen, M.B. Jørgensen, G. Sjøgaard, The effect of worksite physical activity intervention on physical capacity, health, and productivity: a 1year randomized controlled trial, Journal of Occupational and Environmental Medicine 51 (2009), 759-769.

[38]E. Rota, A. Evangelista, G. Ciccone, L. Ferrero, A. Ugolini, M Ceccarelli, C. Galassi, F. Monjini, Effectiveness of an educational and physical program in reducing accompanying symptoms in subjects with head and neck pain: a workplace controlled Trial, The of Journal Headache and Pain 12 (2011), 339-345.

[39]B. Silverstein and R. Clark, Interventions to reduce workrelated musculoskeletal disorders, Journal of Electromyography and Kinesiology 14 (2004), 135-152

[40]T. Shinozaki, E. Tano, K. Murata, Intervention for prevention of low back pain in japanese forklift workers, American Journal of Industrial Medicine 40 (2001), 141-44..

[41]T. Sjögren, K.J. Nissinen, S.K. Järvenpää, M.T. Ojanen, H. Vanharanta, E.A. Mälkiä, Effects of a workplace physical exercise intervention on the intensity of headache and neck and shoulder symptoms and upper extremity muscular strength of office workers: a cluster randomized controlled cross-over trial, Pain 116 (2005), 119-128.

[42]T. Sjögren, K.J. Nissinen, S.K. Järvenpää, M.T. Ojanen, H. Vanharanta, E.A. Malkia, Effects of a workplace physical exercise intervention on the intensity of low back symptoms in office workers: a cluster randomized controlled cross-over design. Journal of Back and Musculoskeletal Rehabilitation. 19 (2006), 13-24.

[43]E. Skargren and B. Öberg, Effects of an exercise program on musculoskeletal symptoms and physical capacity among nursing staff, Scandinavian Journal of Medicine \& Science in Sports 6 (1996), 122-30.

[44]E.P. Takala, E. Viikari-Juntura, E.M. Tynkkynen, Does group gymnastics at the workplace help in neck pain? A controlled study, Scandinavian Journal of Rehabilitation Medicine 26 (1994), 17-20.

[45] J.Y. Tsauo, H.Y. Lee, J.H. Hsu, C.Y. Chen, C.J. Chen. Physical exercise and health education for neck and shoulder complaints among sedentary workers. Journal of Rehabilitation Medicine 36 (2004), 253-257.

[46] T.H. Tveito and H.R. Eriksen, Integrated health programme: a workplace randomized controlled trial. Journal of Advanced Nursing 65 (2008), 110-119.

[47] S.G. van den Heuvel, M.P. de Looze, V.H. Hildebrandt, K.H. Thé, Effects of software programs stimulating regular breaks and exercises on work-related neck and upper-limb disorders. Scandinavian Journal of Work, Environmental \& Health. 2003;29(2):106-16.

[48] M.N. van Poppel, B.W. Koes, T. Smid, L.M. Bouter, A systematic review of controlled clinical trials on the prevention of back pain in industry. Occupational and Environmental Medicine 54 (1997), 841-847.

[49]A.P. Verhagen, H.C. de Vet, R.A.de Bie, A.G. Kessels, M. Boers, L.M. Bouter, et al., The Delphi list: a criteria list for quality assessment of randomized clinical trials for conducting systematic reviews developed by Delphi consensus. Journal of Clinical Epidemiology 51 (1998), 1235-1241.

[50]R.H. Westgaard and J. Winkel, Ergonomic intervention research for improved musculoskeletal health: a critical review. International Journal of Industrial Ergonomics 20 (1997), 463500 . 